\title{
Competencia y compartimiento emocional en usuarios de cocaína: Psicometría y relación con variables sociodemográficas y clínicas
}

\author{
Beatriz A. Corona-Figueroa, ${ }^{1}$ Rebeca Robles-García, ${ }^{2}$ Rosa M. Meda-Lara, ${ }^{3}$ Ana Fresán Orellana, ${ }^{4}$ \\ José Andrés Horacio Cruz-Maycott ${ }^{5}$
}

\section{SUMMARY}

\section{Introduction}

Current evidence suggests a relationship between emotional factors and drugs consumption, although at least both emotional competence and social magazine of emotions have not been studied in cocaine users, in part due to the recent generation of tools for their measure.

\section{Objective}

To evaluate the psychometric properties of the Toronto Alexithymia Scale (TAS), Emotional Competence Questionnaire (ECQ), and the Questionnaire about Beliefs in the effects of the Social Magazine of Emotions (QSME) in Mexican cocaine users, and to establish its relationship with socio-demographic and clinical variables.

\section{Method}

All instruments, as well as the Positive Affectivity (PA) and Negative Affectivity (NA) Scales, were completed twice by 158 cocaine users.

\section{Results}

All scales showed alpha coefficients greater than .70. The TAS and $E C Q$ presented high temporal stability. NA was positively associated with TAS, and negatively with ESQ. PA showed a negative correlation with TAS, and a positive relation with ECQ and QSME. 50.9\% of the patients had a relevant difficulty in identifying and expressing their emotions, which was negatively correlated with schooling.

\section{Discussion and conclusion}

A systematic evaluation and treatment of difficulties in the expression of emotions in cocaine users seems to be necessary. The Spanish versions of the scales are valid and reliable for their use with clinical and research purposes in Mexican cocaine users.

Key words: Alexithymia, emotional competence, cocaine, education, assessment.

Artículo original

\section{RESUMEN}

Introducción

Existe evidencia de relación entre factores emocionales y el consumo de drogas. Sin embargo, al menos la competencia emocional y el compartimiento social de las emociones en usuarios de cocaína no han sido estudiados con anterioridad, en parte debido a la reciente generación de las herramientas para su medición.

\section{Objetivo}

Evaluar las características psicométricas de la Escala de Alexitimia de Toronto (EAT), el Cuestionario de Competencias Emocionales (CEMO), y el Cuestionario de Creencias sobre los efectos de Compartimiento Social de Emociones (CCSE) en pacientes mexicanos usuarios de cocaína, así como establecer su relación con variables socio-demográficas y clínicas.

\section{Método}

Los instrumentos fueron completados junto con las Escalas de Afectividad Positiva (AP) y Negativa (AN) en dos ocasiones por 158 pacientes usuarios de cocaína.

\section{Resultados}

Las tres escalas mostraron coeficientes alpha superiores a .70. La EAT y el CEMO presentaron alta estabilidad temporal a quince días. La AN se asoció positivamente con la EAT y negativamente con el CEMO. La AP se correlacionó negativamente con la EAT y positivamente con el CEMO y el CCSE. El $50.9 \%$ de los pacientes presentaron una dificultad relevante en la identificación y la expresión de sus emociones, que se relacionó de forma negativa con la escolaridad.

\section{Discusión y conclusión}

Se sugiere necesaria la sistematización de la evaluación y el tratamiento de las dificultades en la expresión emocional de usuarios de cocaína. Las versiones en español de las escalas evaluadas en este estudio resultaron válidas y confiables para su uso clínico y de investigación en pacientes mexicanos usuarios de cocaína.

Palabras clave: Alexitimia, competencia emocional, cocaína, escolaridad, evaluación.

Facultad de Psicología, Universidad Autónoma de Guadalajara, México.

Dirección de Investigaciones Epidemiológicas y Psicosociales. Instituto Nacional de Psiquiatría Ramón de la Fuente Muñiz.

Centro Universitario de Ciencias de la Salud, Universidad de Guadalajara, México.

Subdirección de Investigaciones Clínicas. Instituto Nacional de Psiquiatría Ramón de la Fuente Muñiz.

Servicio de Psiquiatría, Hospital Español de México IAP.

Correspondencia: Dra. Rebeca Robles-García. Dirección de Investigaciones Epidemiológicas y Psicosociales, Instituto Nacional de Psiquiatría Ramón de la Fuente Muñiz. Calz. México-Xochimilco 101, San Lorenzo Huipulco, Tlalpan, 14370, México, DF., México. Tel. (55) 4160 - 5143. E-mail: reberobles@imp.edu.mx

Recibido primera versión: 23 de septiembre de 2013. Segunda versión: 1 de septiembre de 2014. Aceptado: 21 de abril de 2015. 


\section{INTRODUCCIÓN}

Los resultados de la más reciente Encuesta Nacional de Adicciones indican que el consumo de drogas ilegales ha aumentado de un $4.6 \%$, observado en 2002 , a un $5.2 \%$ en $2008 .{ }^{1}$ Por grupos de población, siguen siendo los hombres quienes más las consumen (en una proporción de 4.6 hombres por cada mujer). Por droga, el aumento en el consumo de cocaína fue el mayor, pasando de $1.2 \%$ en 2002 a $2.4 \%$ en $2008 .{ }^{1}$ La identificación de los factores psicosociales relacionados con esta problemática puede contribuir a incrementar la efectividad de los esfuerzos encaminados a su prevención y tratamiento.

Entre los factores personales asociados al consumo de drogas y otros comportamientos de riesgo destacan los de tipo emocional. La alexitimia, definida en la década de los 1970 por Sifneos ${ }^{2}$ como la incapacidad para identificar y expresar verbalmente las emociones, es un rasgo presente hasta en el $50 \%$ de los consumidores de drogas, ${ }^{3,4}$ particularmente de cocaína, ${ }^{5}$ y se encuentra asociado con conductas riesgosas, como conducir a exceso de velocidad y el juego compulsivo. ${ }^{6,7}$ Los individuos con alexitimia tienden a enfocarse en sucesos externos en lugar de hacerlo en experiencias internas, suelen carecer de sentido del humor y experimentan falta de sentido con respecto a la vida, ${ }^{8}$ lo que explica en buena medida sus altos índices de afectos negativos, particularmente de depresión y ansiedad., ${ }^{9,10}$ En los estudios de factores socio-demográficos asociados se le ha vinculado repetidamente con bajos niveles de escolaridad, así como socioeconómicos. ${ }^{11,12}$

Los usuarios de cocaína presentan también carencias en el manejo de otras emociones negativas, como la ira y la hostilidad, además de deficiencias importantes en las habilidades sociales, ${ }^{13}$ que podrían estar relacionadas con la alexitimia y/o la carencia de otras competencias emocionales. Picardi et al. ${ }^{14}$ demostraron que la dificultad para verbalizar emociones, por ejemplo, se asoció con ciertos rasgos de personalidad que denotan problemas significativos para ser cálido, cooperativo, sensible y sociable.

\section{Evaluación de la competencia y comparti- miento emocional}

Los primeros métodos para evaluar la capacidad de identificación y la expresión emocional fueron desarrollados por el propio Sifneos, ${ }^{15,16}$ sin embargo, carecían de validez. ${ }^{17,18}$ Fue hasta los 1980 que se desarrolló el instrumento que hoy constituye el estándar de medición de este constructo: la escala de Alexitimia de Toronto (EAT), ${ }^{19,20}$ integrada por tres dimensiones (subescalas): dificultad para identificar emociones, dificultad para describir emociones y pensamiento orientado al exterior. En virtud de sus adecuadas propiedades psicométricas, ha sido traducida a más de 20 idiomas.

De manera más reciente, se han desarrollado otras escalas para evaluar un rango más amplio de competencias emocionales, incluyendo la identificación y la expresión de sentimientos (que implica la alexitimia). Tal es el caso del Cuestionario de Competencia Emocional (CEMO), ${ }^{21}$ que evalúa, además de la discriminación y la expresión verbal de las emociones, una dimensión denominada "empatía". Ésta refleja la capacidad para comprender y conocer las emociones de quienes están alrededor. La competencia emocional ha demostrado una relación positiva con la autoestima, el manejo adecuado de las propias emociones, la resistencia ante las situaciones adversas y el bienestar subjetivo. ${ }^{21}$

Finalmente, otro de los instrumentos complementarios para la evaluación de la expresión emocional es el Cuestionario de Creencias sobre el Compartimiento Social de las Emociones (CCSE): ${ }^{22}$ el proceso en el cual el sujeto describe a un interlocutor un suceso que ha implicado una respuesta emocional. ${ }^{23}$ Las dimensiones del CCSE dan cuenta de las creencias sobre los beneficios intra e interpersonales de expresar o no las emociones. De acuerdo con Páez, Martínez y Rimé, ${ }^{24}$ entre los efectos positivos del compartimiento social de las emociones, destacan la percepción de mayor apoyo social, la disminución de la soledad y la integración social.

Sin embargo, la competencia emocional y el compartimiento social de las emociones son características de la expresión emocional que no han sido estudiadas con anterioridad en los usuarios de cocaína; quizá debido, en parte, a la reciente generación de las herramientas para su medición.

Los objetivos del presente estudio fueron evaluar la consistencia interna, la estabilidad temporal y la validez convergente y divergente con afectividad positiva y negativa, de las versiones españolas de la EAT, el CEMO y el CCSE en pacientes mexicanos usuarios de cocaína. Igualmente, establecer su relación con variables socio-demográficas y clínicas. En congruencia con la bibliografía relacionada, se hipotetizó su asociación con la escolaridad de los pacientes, y se exploró por primera vez su relación con el tiempo bajo tratamiento de la adicción a la sustancia.

\section{MÉTODO}

Se realizó un estudio transversal analítico en las unidades operativas de los Centros de Integración Juvenil (CIJ) de la Zona Metropolitana de Guadalajara, entre diciembre de 2007 y febrero de 2009.

\section{Sujetos}

Se incluyeron usuarios de cocaína con abuso o dependencia, de acuerdo con los criterios diagnósticos de la Asociación Psiquiátrica Americana (DSM-IV), * que acudieron a trata-

\footnotetext{
* El abuso de cocaína es un patrón desadaptativo del consumo de esta sustancia, que genera deterioro y malestar clínicamente significativo por un periodo mínimo de 12 meses, en los que pueden presentarse situaciones como el consumo recurrente de la sustancia. Lo anterior da lugar al incumplimiento de otras obligaciones o causa peligro, problemas legales o sociales y que
} 
miento psicológico, farmacológico y/o de rehabilitación del consumo de sustancias (y los problemas relacionados), con capacidad de leer y escribir, y cuyo estado mental les permitió comprender y responder las escalas autoaplicables.

La mayoría de los pacientes empleaba otras sustancias además de la cocaína (en orden de frecuencia: tabaco, alcohol, marihuana y solventes inhalables). En el estudio participaron sólo aquellos en quienes la cocaína fue la droga de mayor impacto. Esto se documentó mediante la entrevista inicial llevada a cabo por el personal clínico de cada uno de los centros de integración juvenil a donde acudieron los pacientes para ser atendidos. El formato de entrevista inicial consigna el motivo de consulta y los problemas asociados, la situación laboral, escolar y social, y el patrón de consumo de cada una de las sustancias utilizadas con la finalidad de detectar la edad de inicio, frecuencia de consumo, dosis utilizadas y la sustancia que el propio paciente considera como la droga de mayor impacto o que le ha causado mayores problemas.

No se incluyeron los pacientes con algún trastorno psicótico u orgánico cerebral, con intoxicación por cocaína o con síntomas activos de abstinencia.

\section{Instrumentos}

Cuestionario ad hoc de variables sociodemográficas y clínicas. Estas incluyen sexo, edad, estado civil, nivel de estudios, edad de inicio de consumo, vía de administración, tipo y tiempo de tratamiento (farmacológico, psicoterapéutico y/o de rehabilitación, indicado para el abandono o reducción del consumo de sustancias, ya fuera en consulta externa o en tratamiento residencial).

Escala de Alexitimia de Toronto (EAT-20). ${ }^{25}$ La EAT-20 está compuesta por 20 reactivos con opción de respuesta en una escala tipo Likert del 1 al 5 ("totalmente de acuerdo" a "totalmente en desacuerdo"); cinco reactivos están planteados de manera inversa para evitar el fenómeno de aquiescencia. La puntuación total puede oscilar entre 20 y 100 puntos; se sugiere un punto de corte de 61 en población de habla hispana. ${ }^{26}$ La escala ha sido traducida a diferentes idiomas y evaluada psicométricamente en una gran variedad de contextos. ${ }^{27}$ La versión en español, que se ha aplicado en España y diversos países de Latinoamérica, ${ }^{26}$ ha demostrado una adecuada consistencia interna (alphas de .78 para los varones y .85 para las mujeres), ${ }^{27}$ estabilidad temporal a tres semanas $(r=.77)$, y validez de constructo, de criterio ${ }^{28}$ En México se obtuvo un coeficiente alpha de Cronbach incluso mayor (0.87) y se demostró que todos los reactivos discriminan adecuadamente entre sujetos con puntaje alto y aquellos con puntaje bajo (validez de reacitvo). ${ }^{29}$

persisten a pesar de dichos problemas. La dependencia a la cocaína se caracteriza por la presencia de tolerancia o necesidad de ingerir mayores cantidades de la sustancia para conseguir el efecto deseado y por la presencia de abstinencia, síndrome caracterizado por síntomas desagradables de diversa índole, que intentan paliarse con el consumo de la misma sustancia o una similar (Asociación Psiquiátrica Americana. Manual Diagnóstico y Estadístico de los Trastornos Mentales, p. 187).
Cuestionario de Competencia Emocional (CEMO). ${ }^{21}$ El CEMO consta de 12 reactivos con respuestas tipo likert del 1 al 5; cuatro de ellos están redactados de manera inversa. El puntaje mínimo de la escala es de 12 puntos y el mayor es de 60. Puntajes altos representan la capacidad del sujeto para discriminar sus emociones, expresarlas verbalmente, así como para comprender y conocer las emociones de quienes están a su alrededor. Su evaluación psicométrica en población española (473 enfermeras y estudiantes de enfermería de diferentes hospitales de Madrid; 89.6\% mujeres de 27 años como edad promedio) demostró que se trata de una medida válida y con adecuada consistencia interna en las tres dimensiones que evalúa (alpha de .82 para el factor $e x$ presión verbal de las emociones, .75 para el factor discriminación emocional y .72 para el de empatía). ${ }^{21}$

Cuestionario de Creencias sobre los Efectos del Compartimiento Social de las Emociones (CCSE). ${ }^{22}$ El CCSE es un cuestionario de 31 reactivos con opciones de respuesta tipo Likert del 1 al 5; 12 reactivos están planteados en sentido inverso. Posee tres factores: 1) beneficios intrapersonales de compartir las emociones, 2) beneficios de la no expresión social de las emociones y 3) beneficios interpersonales al compartir las emociones. Su puntuación mínima es de 31 y la máxima posible es de 155. Las puntuaciones altas indican actitudes favorables hacia el compartir las vivencias emocionales con los demás. El estudio de la adaptación española de la escala (en 390 estudiantes de la Universidad de Murcia, hombres y mujeres entre 17 y 29 años de edad) mostró que se trata de una medida válida, con adecuada consistencia interna (alpha de .77) y estabilidad temporal a siete semanas $(r=.72){ }^{22}$

Escalas de Afecto Positivo y Negativo-versión para México (PANAS). ${ }^{29}$ Evalúa dos dimensiones bien diferenciadas del afecto: el afecto positivo y el afecto negativo, con base en 20 reactivos; 10 para cada dimensión. Puede aplicarse con diferentes instrucciones temporales para evaluar el constructo como estado (ejemplo: en la última semana) o como rasgo (ejemplo: generalmente). Los reactivos consisten en palabras que describen diferentes sentimientos y emociones a las que la persona califica de acuerdo con el grado en que las experimenta, en una escala de cinco puntos, en donde 1 significa "muy poco o nada", y 5 "extremadamente". Así, el puntaje máximo para cada subescala es de 50. La versión en español para México demostró validez factorial, alta consistencia interna (alphas de .86 a .90 para afecto positivo como estado y como rasgo, y de .84 a .87 para el afecto negativo como estado y como rasgo), estabilidad temporal a dos semanas $(.71$ y .68 para afectividad positiva y negativa como rasgos). ${ }^{30}$ En el presente estudio se aplicó la medición como rasgo (generalmente) tanto en el caso de la afectividad positiva como en la negativa.

\section{Procedimiento}

El estudio fue aprobado por el comité local de Investigación y de Ética. Los pacientes que aceptaron participar de ma- 
nera voluntaria y anónima firmaron el consentimiento bajo información. La aplicación de los instrumentos se llevó a cabo en una sola sesión de aproximadamente 30 minutos. A los quince días se volvieron a aplicar únicamente las escalas sujetas a evaluación psicométrica.

\section{Análisis estadístico}

Los datos fueron capturados y analizados en el paquete estadístico SPSS para Windows, P.C., versión 19. Para la descripción de la muestra se calcularon medias, desviaciones estándar y rangos para variables continuas, así como frecuencias y porcentajes para las categóricas. La consistencia interna se calculó con base en los coeficientes alpha de Cronbach. Como medidas de asociación, se emplearon correlaciones de Pearson; para la comparación de dos medias, se utilizaron pruebas $t$-Student de muestras independientes, y para determinar diferencias entre más de dos grupos, se empleó el análisis de varianza de una vía (ANOVA). El nivel alfa prefijado fue de $95 \%(p \leq .05)$.

\section{RESULTADOS}

Se incluyeron 158 pacientes; la mayoría hombres $(n=140$, $88.6 \%)$ y $\sin$ pareja $(n=80,50.6 \%)$, con una edad promedio de 29.3 años (DS = 9.07) y una escolaridad promedio de 9.6 años $(D S=0.09)$, correspondiente al primer semestre de bachillerato. Las características demográficas, de consumo y de tratamiento se detallan en el cuadro 1.

\section{Confiabilidad y validez}

Las tres escalas mostraron coeficientes alpha de Cronbach superiores a .70. La Escala de Alexitimia de Toronto y el Cuestionario de Competencia Emocional se caracterizaron también por altos coeficientes de correlación entre la primera y la segunda aplicación, no así el Cuestionario de Creencias sobre el Compartimiento Social de Emociones (cuadro 2).

El puntaje promedio de afectividad negativa (26.09 \pm 9.26 , rango $=9-45$ ) se correlacionó positivamente con el total en la Escala de Alexitimia, y en sentido inverso con el Cuestionario de Competencia Emocional. Asimismo, el promedio de afectividad positiva $(33 \pm 9.08$, rango $=10-50)$ se correlacionó negativamente con la puntuación en la Escala de Alexitimia, y de forma positiva con los totales de los cuestionarios de Compartimiento Social de la Emociones y de Competencia Emocional.

\section{Competencia y compartimiento emocional en usuarios de cocaína: Relación con variables sociodemográficas y clínicas}

El cuadro 2 presenta las puntuaciones promedio de los participantes en las tres escalas de competencia y compartimien-
Cuadro 1. Características demográficas y clínicas $(n=158)$

\begin{tabular}{|c|c|c|}
\hline & $n(\%)$ & $\bar{x}+D S$ \\
\hline \multicolumn{3}{|l|}{ Sexo } \\
\hline Hombres & $140(88.6)$ & \\
\hline Mujeres & $18(11.4)$ & \\
\hline Edad (años) & & $29.30 \pm 9.07$ \\
\hline \multicolumn{3}{|l|}{ Estado civil } \\
\hline Sin pareja & $80(50.6)$ & \\
\hline Con pareja & $78(49.4)$ & \\
\hline Escolaridad (años) & & $9.60 \pm 0.09$ \\
\hline Edad de inicio del consumo & & $21.86 \pm 4.35$ \\
\hline \multicolumn{3}{|l|}{ Vía de administración } \\
\hline Inhalada & $74(46.8)$ & \\
\hline Fumada & $78(49.4)$ & \\
\hline Otra & $6(3.8)$ & \\
\hline \multicolumn{3}{|l|}{ Tipo de tratamiento } \\
\hline Hospitalario & $62(39.2)$ & \\
\hline Consulta externa & $96(60.8)$ & \\
\hline \multicolumn{3}{|l|}{ Tiempo en tratamiento } \\
\hline Rango 1: Menos de un mes & $108(68.3)$ & \\
\hline Rango 2: De uno a tres meses & $32(20.2)$ & \\
\hline Rango 3: De tres a 11 meses & $5(3.2)$ & \\
\hline Rango 4: De uno a tres años & $8(5.1)$ & \\
\hline Rango 5: Más de tres años & $5(3.2)$ & \\
\hline
\end{tabular}

to emocional. De acuerdo con el punto de corte propuesto para la escala de Alexitimia, el 50.9\% $(n=55)$ de los casos presentaron una puntuación alta (superior a 60 puntos), denotando así una dificultad clínicamente relevante tanto en la identificación como en la expresión de sus emociones.

La alexitimia se correlacionó de forma negativa con la escolaridad de los pacientes $(r=-.23, p \leq .05)$; por su parte, la Competencia emocional se relacionó de manera positiva con su edad cronológica $(r=.22, p \leq .01)$.

Además, al menos dos de las medidas difirieron significativamente entre pacientes ubicados en distintos rangos de tiempo en tratamiento* (Competencia emocional: $F=2.862$, $p=.017$; Creencias sobre los efectos del compartimiento social de emociones: $F=3.079, p=.013$ ). Los pacientes con mayor tiempo en tratamiento presentaron puntuaciones más altas tanto de competencia emocional (Rango 1:36.54 \pm 8.26, Rango 2:40.17 \pm 8.13. $t=-2.006, g l=49.39, p=.05)$ como de creencias de los efectos positivos (o beneficios) del compartimiento social de las emociones (Rango 1:107 \pm 15.53, Rango 5:131 $\pm 4.08, t=-1.58, g l=13.84, p \leq .0001)$.

\section{DISCUSIÓN Y CONCLUSIÓN}

Los objetivos del presente estudio incluyeron la evaluación psicométrica de tres instrumentos para medir compe-

\footnotetext{
* Rangos de tiempo en tratamiento: Fase diagnóstica; menos de un mes; de uno a tres meses; de tres a seis meses; de seis meses a un año; de uno a tres años; más de tres años.
} 
Cuadro 2. Puntuaciones totales, consistencia interna, estabilidad temporal y validez convergente y divergente de las medidas de competencia y compartimiento emocional

\begin{tabular}{lccccc}
\hline Escala & Media \pm DS (rango) & Alpha & Test-Retest & $r$ Pearson AP* $r$ Pearson AN* \\
\hline Escala de Alexitimia de Toronto & $59.38 \pm 15.29(24-91)$ & .84 & .75 & $-.35, p \leq .01$ & $.54, p \leq .01$ \\
$\begin{array}{l}\text { Cuestionario de Creencias sobre el Com- } \\
\text { partimiento Social de las Emociones }\end{array}$ & $110.54 \pm 15.14(60-143)$ & .84 & .57 & $.23, p \leq .05$ & $-.13, N^{*}$ \\
Cuestionario de Competencia Emocional & $37.99 \pm 8.00(16-57)$ & .74 & .77 & $.24, p \leq .01$ & $-.20, p \leq .05$ \\
\hline
\end{tabular}

* AP: Afectividad positiva; AN: Afectividad negativa; NS: No significativa.

tencias emocionales. Básicamente, las relacionadas con la identificación y expresión de emociones. También, explorar su relación con variables sociodemográficas y clínicas, especialmente con la escolaridad y el tiempo en tratamiento, respectivamente.

De acuerdo con los resultados, las versiones en español de las tres escalas son válidas y confiables para su uso con fines clínicos y de investigación en pacientes mexicanos usuarios de cocaína. Sus puntuaciones totales correlacionaron en la dirección esperada con las medidas de afectividad positiva $(\mathrm{AP})$ y negativa (AN) como rasgos: ${ }^{8,9,13}$ la $\mathrm{AN}$ se asoció positivamente con la Escala de Alexitimia de Toronto (EAT20), y en sentido inverso con el Cuestionario de Competencia Emocional (CEMO); la AP se correlacionó negativamente con la EAT-20 y de forma positiva con el CEMO y con el Cuestionario de Creencias sobre los efectos (positivos) del Compartimiento Social de Emociones (CCSE).

Además, los coeficientes de consistencia interna y estabilidad temporal de las tres escalas resultaron francamente similares a los reportados para sus versiones españolas. ${ }^{21,22,27,28}$ Psicométricamente hablando, la EAT-20 resultó también la más adecuada, pues además de tener una alta consistencia interna (al igual que el CCSE), se caracteriza igualmente por una adecuada estabilidad temporal (similar a la encontrada para el CEMO). En cambio, el CCSE tiene índices moderados de estabilidad temporal a dos semanas, y el CEMO, una menor consistencia interna.

De tal manera, cuando el objetivo clínico o de investigación sea la sola evaluación de la alexitimia, la EAT-20 se mantiene como la medida más recomendada, también en pacientes mexicanos. Sin embargo, el CEMO ofrece la ventaja de evaluar, además de la competencia para identificar y expresar emociones, la dimensión denominada "empatía", referente a la capacidad para comprender y conocer las emociones de los demás (no sólo las propias). La evaluación de esta capacidad podría ser relevante en muchos de los pacientes usuarios de drogas que reportan interacciones sociales negativas y son considerados para recibir entrenamiento en habilidades sociales. ${ }^{13}$ Aunque la EAT mostró mayor confiablidad, los índices de consistencia interna y estabilidad temporal del CEMO son adecuados.

Por su parte, el CCSE es una medida con alta consistencia interna que complementaría la evaluación de los pacientes si se desea conocer el peso de las variables cognitivas específicas en la disposición para compartir emociones: las creencias acerca de los beneficios intrapersonales que conlleva hacerlo o no hacerlo. ${ }^{22}$ Esto permitiría dirigir esfuerzos terapéuticos para modificar tales percepciones, aunque la evaluación de la efectividad de este tipo de intervenciones, por medio de la escala, se vería limitada en cuanto a su moderada estabilidad temporal.

En cuanto a la relación entre estas variables de competencia emocional y las sociodemográficas y clínicas, en el presente estudio se corroboró la asociación inversa con la escolaridad de los pacientes planteada en algunas investigaciones antecedentes. ${ }^{10}$ En el presente trabajo, en tanto mayor puntuación de alexitimia, se registró una menor escolaridad, lo que sugiere que la educación formal puede proporcionar a los pacientes mayores elementos para que identifiquen y verbalicen sus emociones.

Adicionalmente, se obtuvo evidencia de relación entre la competencia emocional y la edad cronológica de los pacientes (que se asociaron de forma positiva y estadísticamente significativa); así como de mayores puntuaciones, tanto en el CEMO como en el CCSE, entre los pacientes con más tiempo bajo tratamiento. Lo anterior da cuenta de las posibilidades de aprendizaje de las competencias emocionales por parte de los pacientes.

Así, aunque el presente estudio tiene limitaciones importantes, especialmente en relación con la poca participación de mujeres (lo que impidió comparaciones por sexo) y con el tipo de muestreo no aleatorio (que exige cautela en la generalización de resultados), proporciona evidencia de la utilidad específica de tres escalas de medición de competencias emocionales relacionadas con la identificación y la expresión emocional. Se propone su uso con fines clínicos y de investigación en pacientes mexicanos usuarios de cocaína, muchos de los cuales (prácticamente la mitad de acuerdo con los datos de este estudio) pueden presentar dificultades emocionales importantes (sobre todo los más jóvenes y de menor escolaridad), que son susceptibles de mejora mediante un tratamiento.

Finalmente, es posible sugerir la utilidad de fomentar tanto la identificación como la expresión de emociones propias, así como el reconocimiento de las emociones de los demás y de la importancia de su compartimiento social, como parte de los esfuerzos de prevención de las adicciones y la promoción de la salud mental en general. 


\section{Financiamiento}

Ninguno.

\section{Conflictos de interés}

Los autores declaran no tener ningún conflicto de interés.

\section{Agradecimientos}

De manera muy especial a las autoridades de los Centros de Integración Juvenil por la autorización del proyecto y por el apoyo logístico para su implementación: a la licenciada Carmen Fernández Cáceres, a los doctores Ricardo Sánchez Huesca, David Bruno Díaz Negrete y Luis Solís Rojas, al Coordinador Regional Zona Occidente de Centros de Integración Juvenil, maestro Enrique de Jesús Aceves Arce, y a los Directores de las Unidades Operativas donde se evaluó a los pacientes: a los doctores Víctor Manuel Márquez Soto y Guillermo Rochin Güereña, a los licenciados Ana Lucía Álvarez Tamayo, José G. Gómez Martínez, Luis Gómez Villaseñor, y al doctor Alfredo Rizo Méndez; a los asesores externos y autores también de los instrumentos doctores Darío Páez, Francisco Martínez, Bernardo Moreno, Leticia Guarino, Gloria García de la Banda; y al psicólogo Dagoberto Monjarás Terrero, por su apoyo informático.

\section{REFERENCIAS}

1. Secretaría de Salud. Encuesta Nacional de Adicciones 2008. Ciudad de México: 2008.

2. Martínez F, Ortiz B. La evaluación de la alexitimia. En: Páez D, Casullo M (eds.). Cultura y alexitimia. Buenos Aires: Paidós; 2000.

3. Casullo M, Castro A, D' Anna A. Alexitimia y características de personalidad de jóvenes adictos. En: Páez D, Casullo M (eds.). Cultura y alexitimia. Buenos Aires: Paidós; 2000.

4. Gómez-Fernández A, Espina-Eizaguirre A. Alexitimia y características clínicas en el abuso de opiáceos. Revista Toxicodependencias 1997;3:77-85.

5. Chiang-Shan R. Alexithymia and stress-induced brain activation in cocaine-dependent men and women. J Psychiatry Neurosci 2000;31:115121.

6. Lumley MA, Roby KR. Alexithymia and pathological gambling. Psychother Psychosom 1995;63:201-206.

7. Sivak R, Wiater A. Alexitimia, la dificultad para verbalizar afectos. Buenos Aires: Paidós; 2000.

8. Pérez-Rincón $\mathrm{H}$. Alexithymia considered as a survival of an archaic structure of language. Importance of Bruno Snell's theories. New Trends Exp Clin Psychiatr 1997;13:159-160.

9. Le HN, Ramos MA, Muñoz RF. The relationship between alexithymia and perinatal depressive sympthomatology. J Psychosom Res 2007;62:215-222.

10. Devine H, Stewart SH, Watt MC. Relations between anxiety sensitivi- ty and dimensions of alexithymia in young adult sample. J Psychosom Res 1999;47:145-158.

11. Salminen JK, Saarijärvi S, Äärelä E, Toikka T et al. Prevalence of alexithymia and its association with sociodemographic variables in the general population of Finland. J Psychosom Res 1999;46:75-82.

12. Franz M, Popp K, Schaefer $R$, Sitte $W$ et al. Alexithymia in the German general population. Soc Psychiatry Psychiatr Epidemiol 2008;43:54-62.

13. Beck AT, Wright FD, Newman CF, Liese BS. Terapia Cognitiva de las drogodependencias. Barcelona: Paidós; 1999.

14. Picardi A, Toni A, Caroppo E. Stability of alexithymia and its relationships with the 'big five' factors, temperament, character, and attachment style. Psychother Psychosom 2005;74:371-378.

15. Sifneos P. The prevalence of "alexithymic" characteristics in psychosomatic patients. Psychother Psychosom 1973;22:255-262.

16. Apfel RJ, Sifneos PE. Alexithymia: concept and measurement. Psychother Psychosom 1979;32:180-190.

17. Gardos G, Schniebolk S, Mirin SM, Volk PC et al. Alexithymia: towards validation and measurement. Compr Psychiatry 1984;25:278-282.

18. Bagby RM, Taylor GJ, Atkinson L. Alexithymia -a comparative-study of three self-report measures. J Psychosom Res 1988;32:107-116.

19. Bagby R, Parker J, Taylor G. The 20-item Toronto Alexithymia Scale, I: item selection and crossvalidation of the factor structure. J Psychosom Res 1994;38:23-32.

20. Bagby R, Taylor G, Parker J. The 20-item Toronto Alexithymia Scale, II: convergent, discriminant and concurrent validity. J Psychosom Res 1994;38:33-40.

21. Garrosa E, Moreno B, Rodríguez A, Sanz A. La influencia del estrés de rol y la competencia emocional en el desgaste profesional. Información Psicológica 2008:91-92.

22. Martínez-Sánchez F, Zech E, Páez-Rovira D. Adaptación española del cuestionario de creencias sobre los efectos del comportamiento social de las emociones (BSEQ). Ansiedad Estrés 2004;10:63-74.

23. Zech $E$. The effects of the communication of the emotional experience. Tesis doctoral. Bélgica: Facultad de Psicología. Universidad Católica de Lovaina; 2000.

24. Páez-Rovira D, Martínez-Sánchez F, Rimé B. Los efectos del compartimiento social de las emociones sobre el trauma del 11 de marzo en personas no afectadas directamente. Ansiedad Estrés 2004;10:221-232.

25. Taylor GJ, Ryan D, Bagby RM. Toward the development of a new self-report alexithymia scale. Psychother Psychosom 1985;44:191-199.

26. Páez D, Casullo MM. Cultura y alexitimia. Buenos Aires: Paidón; 2000.

27. Páez D, Martínez-Sánchez F, Velasco C, Mayordomo S et al. Validez psicométrica de la Escala de Alexitimia de Toronto (TAS-20): un estudio transcultural. Boletín Psicología 1999;63:55-76.

28. Martínez-Sánchez F, Ortiz B. La evaluación de la alexitimia. Alexitimia Cultura 2000:35-51.

29. Pérez-Rincón H, Cortés J. Ortíz S, Peña J et al. Validación y estandarización de la versión española de la Escala modificada de Alexitimia de Toronto. Salud Ment 1997;20:30-34.

30. Robles R, Páez F. Estudio de traducción al español y propiedades psicométricas de las Escalas de Afectividad Positiva y Negativa (PANAS). Salud Ment 2003;26:69-74. 